\title{
Anal Cancer in a HIV Sero-Positive Bisexual Bachelor-A Case Report and Literature Review
}

\author{
Victor Nwagbara*, M. E. Asuquo, J. A. Ashindoitiang, S. O. Akpan \\ Department of Surgery, University of Calabar, Calabar, Nigeria \\ Email: aikayvic@gmail.com, aikay_en@yahoo.com,*vicnwagbara@unical.edu.ng
}

How to cite this paper: Nwagbara, V., Asuquo, M.E., Ashindoitiang, J.A. and Akpan, S.O. (2018) Anal Cancer in a HIV Sero-Positive Bisexual Bachelor-A Case Report and Literature Review. Case Reports in Clinical Medicine, 7, 601-607.

https://doi.org/10.4236/crcm.2018.712055

Received: October 20, 2018

Accepted: December 15, 2018

Published: December 18, 2018

Copyright ( 92018 by authors and Scientific Research Publishing Inc. This work is licensed under the Creative Commons Attribution International License (CC BY 4.0).

http://creativecommons.org/licenses/by/4.0/

\begin{abstract}
Anal cancer, especially squamous cell carcinoma (SCC) variety, is evolving with rising incidence globally. This is due to the increasing adoption of risky life styles and social habits even in populations with rare occurrences previously. This case report aims to expose risk factors exhibited by a patient recently managed for anal cancer in our facility before his demise. Early exposure to bisexual orientation, receptive anal intercourse and multiple sexual partners, are notable high risk factors for the development of anal SCC. The permissive role of smoking, illicit drug and alcohol use enhances the disease occurrence. Acquisition of human immune-deficiency virus (HIV) sero-positive status further reduces the body's immune competence and enhances early age of onset as well as accelerates development of anal SCC. Proper history exploring the risk factors, physical/anorectal examination and histological analysis, are vital for proper diagnosis and staging which guides appropriate treatment with chemo-radiation as mainstay.
\end{abstract}

\section{Keywords}

Anal Cancer, Bisexual, Human Immune-Deficiency Virus, Sero-Positive Status, Squamous Cell Carcinoma

\section{Introduction}

Anal cancer is a rare disease globally and much rarer in our setting even when symptomatic, though rising in incidence among developed countries [1] [2]. Most cases are seen in the elderly women. Squamous cell carcinoma (SCC) is the predominant type and constitutes up to 80 percent of cases of anal cancer [3]. Human Papilloma Virus (HPV) sero-types 16 and 18 infection, has been impli- 
cated as causative agents in about $84 \%$ of cases [3]. Life styles/social habits such as having multiple gay partners, receptive anal intercourse as well as smoking cigarettes, doing drugs and alcohol abuse, contribute significantly to increase the risk of developing anal SCC [1] [2] [3]. The acquisition of human immunodeficiency virus HIV) seropositivity is an independent risk factor [3] which increases the risk exponentially. Asuquo et al. [4], described SCC irrespective of site, as a slow-growing malignancy that hardly threatens life.

Although gay behaviour is culturally and legally prohibited in our country, an increasing number of people mostly youths continue to engage in it and other risky life styles clandestinely. An appropriate prevention strategy is needed to stem the rising cases of risky sexual behaviour/life style which may lead to higher incidence of anal cancer in the near future through health education.

\section{Case Report}

UMI is a single, 34 year old male Caterer, working in Lagos. He was admitted through Accident/Emergency in July 2016 and later referred to our surgical team on account of anal mass and bleeding per rectum of 6 months duration in addition to general lassitude.

Patient was apparently well until about seven months prior to presentation when he noticed a small, painless, itchy anal mass. The mass gradually increased in size over a few months, became ulcerated and bled intermittently prior to presentation. It was later associated with insidious onset of pain, which was sharp and persistent, non-radiating, aggravated by strenuous activities and defecation. There was no relieving factor but he adopted the prone position as the most comfortable position for most of the day.

The passage of intermittent bloody stool started 2 weeks after noticing the anal mass. The blood occasionally smeared the stool and sometimes came as frank bleeding. It did not contain clots and was not associated with dizziness initially. His stool was soft, formed and regular once daily. Later, he noticed purulent anal discharge which preceded the passage of mal-odorous stool and progressed to passage of dark stool mixed with pus. There was associated loss of appetite and feeling unwell but no nausea nor vomiting. He had no abdominal pain or change in bowel habit. There was associated marked weight loss and progressive body weakness. He presented at a private hospital in Lagos where he was diagnosed of RVD and placed on ARV (Lamivudine, Abacavir, Efavirenz) in addition to receiving one unit of whole blood transfusion. His symptoms did not improve after one month of treatment, thus he relocated to his Calabar home town. He got progressively worse with extreme weakness and presented at the Accident/Emergency unit of a tertiary health facility for further management after defaulting on his ARV drug therapy for six months without explanation.

He had no significant past medical history. He takes alcohol occasionally denies use of tobacco or hard drugs in any form.

He was the $3^{\text {rd }}$ child among 6 siblings in a polygamous setting. He was single 
and had bisexual orientation. He engaged in both oral and receptive anal intercourse (RAI) for about 14 years but decided to stop for no obvious reason a year prior to onset of illness. Hehad five regular male partners over this period whom he claimed became RVD positive and on treatment.

Physical examination showed a chronically ill-looking, cachexic, febrile young man in painful distress. He was conscious, alert and well-oriented. He had severe pallor but was anicteric and dehydrated. Oral thrush was present with bilateral inguinal lymph node enlargement and bilateral pitting pedal oedema. His blood pressure, pulse rate and respiratory rates were within normal range. He had no sensory neurological deficit but power in the lower limbs was grade three. His chest was clinically clear. He had no obvious deformity. The abdomen was not remarkable.

Rectal examination revealed perianal warts with huge ulcerated anal mass close to the midline posteriorly (see Figure 1 ) with associated multiple anal fistulae draining pus and blood. The anal sphincter was lax and adjacent rectal wall had multiple tender indurated nodules extending from the anterior to the posterior aspect and some granular areas, which provoked bleeding on palpation. The prostate gland was clinically normal and the examining fingerstall was stained with fresh blood.

A working diagnosis of RVD (HIV/AIDS) clinical stage IV with Malignant Anorectal Fistula and Anal warts was made and he was subsequently admitted for stabilization and optimization. Initial investigation results showed a low haemogram of $6.4 \mathrm{~g} / \mathrm{dl}$ (Reference Value 13 - $18 \mathrm{~g} / \mathrm{dl}$ ), with anisocytosis, macrocytosis, poikilocytosis and hypochromasia. Total White Blood Cell (WBC) count was $8.5 \times 10^{9} / \mathrm{L}$ (Reference Value $4-11 \times 10^{9} / \mathrm{L}$ ) with relative lymphocytosis. The $\mathrm{CD}^{+} \mathrm{T}$-Cell count was 103 cells/ul (Reference Value $>500 /$ ul). Serum Urea and Electrolytes assay, Liver function test and urinalysis results were within normal range. Random Blood Sugar (RBS) estimation was abnormally high at $16.1 \mathrm{mmol} / \mathrm{l}$ (Reference Value $4.4-8.9 \mathrm{mmol} / \mathrm{L}$ )

Abdominal ultrasound scan, Serum protein assay and Wound swab for microscopy, culture and sensitivity were requested but not done because of financial difficulty.

A wedge biopsy of the ulcerated anal lesions showed a well differentiated Squamous cell carcinoma (SCC) extending to the underlying dermis and areas of dysplastic keratinocytes (see Figure 2).

He was re-commenced on anti-retroviral treatment using Lamivudine/Abacavir/Efavirenz combination plus antibiotics, oral antifungal, haematinics, daily Sitz bath and wound dressing as well as nourishing high protein meals. He also received two units of compatible whole blood transfusion. Exhibition of cytotoxic chemotherapy was with-held pending improvement of haemogram above $10 \mathrm{~g} / \mathrm{dl}$ and CD4 cell count above 200. The above stated line of management was continued but the patient got worse with more copious bloody stool, increasing general lassitude and poor appetite. He remained unstable and was unfit to undergo chemo-radiotherapy until his eventual demise a few weeks later. 


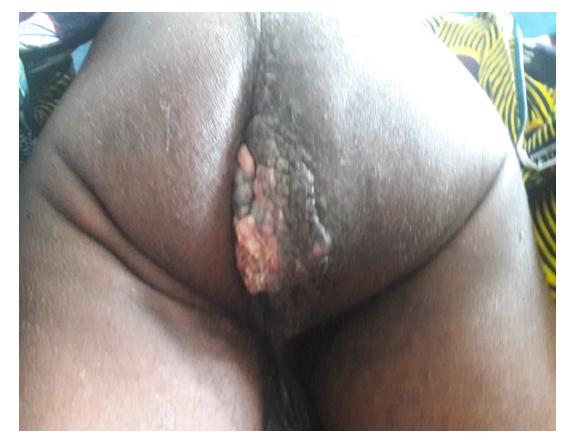

Figure 1. Clinical photograph anal cancer with peri-anal warts.

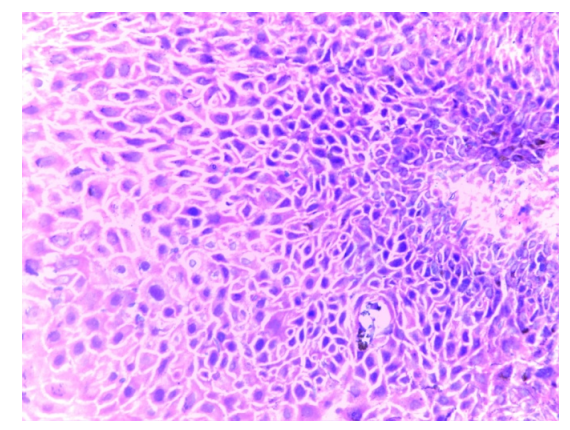

Figure 2. Photomicrograph of anal SCC $(\mathrm{H} \& \mathrm{E} \times 100)$.

\section{Discussion}

Anal cancer constitutes about $1 \%$ of all new cases of cancers diagnosed each year but represents $1.5 \%$ - 2\% of all Gastro-Intestinal Tract cancers with $2 \%$ increase in annual incidence [5] [6]. It occurs mostly as Squamous Cell Carcinoma (SCC) in $80 \%$ of cases and seemed rare before 1990 [1] [3] [5] both locally and globally. SCC has been known as the commonest skin malignancy in Blacks while Basal cell Carcinoma (BCC) predominates in Caucasians [4]. It is mostly found among elderly females but has recently been reported to increase in incidence among young adult males who are HIV sero-positive and/or men who have sex with men MSM) especially in developed countries [1] [6] [7]. Asuquo et al. [4], found ano-rectal and genital SCC as the $3^{\text {rd }}$ commonest site of involvement among Blacks in Nigeria and Tanzania and described SCC as a slow-growing malignancy that hardly threatens life. The rise in incidence is most evident among young people who are HIV positive and men having anal sex with men (MSM) [5] [6] [7]. Reports from United States data [1] [6] [7], shows that among HIV positive population, there was a 40 - 80 fold increase in incidence above the general population, from 19 to 78.2 per 100,000 person-years while among (MSM) having receptive anal intercourse (RAI), anal cancer was diagnosed in 35 per 100,000 person-years, an incidence rate similar to cervical cancer in females, which is also caused by Human Papilloma Virus (HPV) 16 and 18. The contribution of anal SCC to cancer mortality is unknown largely because squamous 
cell carcinoma usually run a slow, chronic course and sufferers hardly die of their cancer [7] [8]. The introduction of Highly Active Anti-Retroviral Treatment (HAART) which ensures a longer life span for HIV positive people will also raise the prevalence of anal cancer. The male/female ratio seems to have reversed with an obvious male predominance as more HIV positive middle-aged men were being seen for a relatively rare disease that typically occur in older women [9]. Due to the increased incidence of SCC of the anus following the discovery of HIV/AIDS and the close association between them, some workers have suggested the inclusion of anal SCC as an AIDS-defining illness nay, severe AIDS [1] [8] [10]. Factors implicated in the aetiology of anal cancer among HIV positive individuals include HPV status especially HPV 16 or 18, sexual habit, in particular receptive anal intercourse in Men having Sex with Men (MSM), multiple sexual partners and smoking. HPV is well known as a sexually transmitted infection (actually the most prevalent STI in USA) that causes anal cancer. The virus resides in outermost layer of skin or mucosa especially in immunosuppressed persons, causing different grades of dysplastic reactions and ultimately invasive SCC [1] [3] [10].

HIV positive persons tend to develop anal cancer (SCC) at an earlier age especially MSM indulging in RAI with multiple partners like our index patient. It has also been noted that among HIV/AIDS patients, those who are homosexual (MSM) or Bisexual account for much of the incidence and mortality with majority succumbing within $10-17$ months [9] [10]. It is also an established fact that Human Papilloma Virus (HPV) infection causes anal warts which is a precursor or high risk factor for development of anal intra-epithelial neoplasia and ultimately anal cancer, notably seen more among those practising receptive anal intercourse [3]. HPV types 16, 18, 33 and 39 in decreasing order of frequency, are the same agents responsible for cervical squamous cell cancer in females. HIV positive persons are more at risk of developing persistent HPV infection than immune-competent individuals due to immunosuppression. This increases their risk of having anal SCC suggesting that immunosuppressed individuals have poor clearance of HPV in their bodies [3] [10]. HPV infection leads to the development of anal intra-epithelial dysplasia or neoplasia (AIN) also called squamous intra-epithelial lesion which may be low-grade (LSIL/LGAIN) or high-grade (HSIL/HGAIN). This slowly leads to anal carcinoma in situ and eventually to invasive anal cancer if not treated as indeed most cases of SCC in Blacks are seen in advanced stage as in our index case. AIN may proceed without any health complaint in those affected. Warty lesions are commonly seen in HPV induced cases [4]. Among MSMs, receptive anal intercourse is the most common risk factor for developing anal cancer while concurrent HIV infection compounds the problem. A history of multiple sex partners, syphilis, hepatitis, smoking, drug and alcohol use increases the risk [8]. The diagnosis of anal cancer is often delayed until it is advanced. It is therefore advised that clinical suspicion should be considered in patients with anal symptoms like itching, anal pain, 
anal bleeding or diarrhoea in addition to visual inspection and digital rectal examination, and capped with biopsy with or without high resolution anoscopy. Annual anal PAP smear is recommended in those at risk as is done to screen for cervical cancer in women [8] [9]. The histologic examination of a biopsy specimen is very essential to differentiate anal SCC from other similarly presenting anal lesions like Melanoma, Adenocarcinoma and Basal Cell Carcinoma.

The treatment of anal cancer is not straight forward. Histologic classification and staging are important. Anal SCC spreads mostly by the lymphatic route. Patient should be fit or optimized for whatever treatment modality in use. The treatment of the various grades of precancerous lesions holds the key to preventing advancement while screening and vaccination of those with risky behaviour has been advocated. This appears feasible since the same approach has reduced the incidence of cervical cancer in women and both anal and cervical cancer share the same aetiological agent in HPV types 16 and 18. Patients with RVD in addition to anal cancer have a peculiar challenge. They usually succumb to Retro-Viral Disease (RVD) rather than anal cancer, from effects of HIV and very low $\mathrm{CD} 4^{+}$count $<200$ [7] [9]. This further suggests that HIV positive patients with anal cancer, cannot withstand the toxicity of chemotherapy and must have intensive anti-retroviral therapy initially, before commencing cancer chemotherapy. The treatment of invasive anal SCC is based on Radiotherapy and Chemotherapy using Mitomycin C (MMC) and 5-Fluoro-Uracil. In addition, combination with HAART will greatly increase toxicity as both are immunosuppressive. A CD $4^{+}$count of 200 or more is used to determine HIV+ patients in whom chemotherapy should be tried [1] [7] [8] [9]. In addition, several workers have noted the difficulty and severity of adverse reactions to cytotoxic therapy in those with HIV/AIDS. They opine that intensive anti-retroviral therapy must be instituted first and continued until $\mathrm{CD}^{+}$count rises above 200 before exhibiting cytotoxic chemotherapy as planned in our index patient before his demise.

In cases of persistent biopsy positive anal cancer after chemo-radiation, with no metastatic lesions, it has been suggested that an Abdomino-Perineal resection with terminal colostomy should be considered [1] [6]. Current studies on treatment of anal SCC is focusing on molecular targeted therapy as some genes like EGFR, c-Met and VEGFR [1] are over-expressed especially in those who are HIV positive. The goal of therapy in anal SCC, even among HIV positive ones is to eradicate the disease before dissemination and improve the quality of life including avoidance of colostomy.

Preventive measures are equally important but it requires a high index of suspicion to identify those with risky life styles as targets for health education, screening and vaccination by using Gardasil and early treatment of precancerous lesions.

Very important risk factors raised in this case need closer attention in the populace with a view to marshalling strategies for curbing or stemming the rising incidence. It also underlies the importance of early detection and prompt treatment before the development of invasive anal SCC. HIV positive patients 
who practice anal intercourse should be encouraged to stop the act, go for regular screening and take their anti-retroviral therapy very serious.

\section{Conflicts of Interest}

The authors declare no conflicts of interest regarding the publication of this paper.

\section{References}

[1] Dandapani, SV. Eaton, M. Thomas Jr., C.R. and Pagnini, P.G. (2010) HIV positive Anal Cancer: An Update for the Clinician. Journal of Gastrointestinal Oncology, 1, 34-44.

[2] Newman, E. (2015) Anal Cancer and HPV: What Do Gay Men Need to Know? http://betablog.org/anal-cancer-hpv-gay-men-need-know

[3] Dekoj, T.R. and Espat, N.J. (2018) Anal Cancer. Medscape.

[4] Asuquo, M.E., Nwagbara, V.I. and Omotoso, J. (2011) Squamous Cell Carcinoma: Clinical Pattern and Challenges of Treatment. European Journal of Plastic Surgery, 34, 459-464. https://doi.org/10.1007/s00238-011-0555-4

[5] Osborne, M.C., Maykel, J., Johnson, E.K. and Steele, S.R. (2014) Anal Squamous Cell Carcinoma: An Evolution in Disease and Management. World Journal of Gastroenterology, 20, 13052-13059. https://doi.org/10.3748/wjg.v20.i36.13052

[6] Salati, S.A. and Al Kadi, A. (2012) Anal Cancer-A Review. International Journal of Health Sciences, 6, 206-230. https://doi.org/10.12816/0006000

[7] Palesky, J. (2009) Human Papillomavirus-Related Disease in People with HIV. Current Opinion in $H I V$ and AIDS, 4, 52-56. https://doi.org/10.1097/COH.0b013e32831a7246

[8] (2000) Poor Outcomes Seen in HIV+ Patients with Anal Cancer. http://www.cancernetwork.com/articles/poor-outcomes-seen-hiv\%20patients-analcancer

[9] Medical Care Criteria Committee (2007) Anal Dysplasia and Cancer Guideline. https://www.hivguidelines.org/mgmlp_media_folder/Anal-Dysplasia-and-Cancer/

[10] Margolies, L. and Goeren, B. (2016) Anal Cancer, HIV and Gay/Bisexual Men. The National LGBT Cancer Network. 